\title{
Ruptured internal iliac artery aneurysm masquerading as an inguinal hernia
}

\begin{abstract}
Internal iliac artery aneurysms are rare, representing less than $2 \%$ of all aneurysms. Nonetheless, the mortality is high at up to $60 \%$ when ruptured. Ruptured internal iliac artery aneurysms commonly present with overt abdominal pain and systemic instability. They can also present with symptoms of pelvic organ dysfunction, such as urinary retention or obstipation. This report describes the first case of a ruptured internal iliac artery (IIA) aneurysm masquerading as a reducible inguinal hernia. It was diagnosed on a Computed Tomography scan, which showed a large contained ruptured right IIA aneurysm with extension of haemorrhage through the hernia sac. He underwent an endovascular procedure to exclude the aneurysm that was uncomplicated, and he had an uneventful recovery. It highlights the importance of considering IIA aneurysms as differentials for abdominal masses, groin lumps or vague symptoms of pelvic organ dysfunction. Thorough exploration of the history and careful examination will help flag these diagnoses in our clinical assessment.
\end{abstract}

Volume II Issue 2 - 202I

\author{
Jia Ying Isaac Tay \\ Department of General Surgery, The Royal Melbourne Hospital, \\ Australia
}

Correspondence: Jia Ying Isaac Tay, Department of General Surgery, The Royal Melbourne Hospital,Australia

Tel +6I 48I 309 0 I2, Email jyisaac.tay@gmail.com

Received: March 01, 202I | Published: March 19, 2021

Keywords: internal iliac artery aneurysm, ruptured aneurysm, endovascular surgery, embolization coil, stent graft, inguinal hernia

\section{Case presentation}

An 80-year-old gentleman presented to Emergency Department with a right groin lump and iliac fossa pain. He presented twice in the preceding 2-days with the same presentation, which was treated as a reducible inguinal hernia and planned for outpatient follow-up.

Our patient presents with a new right groin lump that was mildly tender and manually reducible. He also had right iliac fossa pain, which was severe and of sudden onset 3-days ago, causing him to have a syncope then. This pain had since reduced. There were no other associated features, such as fever, nausea, changes in bowel habits or urination. His medical history consisted only of knee and back osteoarthritis. He had no past or family history of hernias, aneurysms, or connective tissue disease. He was a life-long non-smoker. On examination, he had normal and stable vital signs. There was a small palpable groin lump with cough impulse on standing, which reduced on lying. His lower limb pulses were present, and neurology was normal. He was tender in the right iliac fossa with no peritonism.

$\mathrm{He}$ had normal inflammatory markers, $\mathrm{Hb} 133 \mathrm{~g} / \mathrm{L}$ and Creatinine 104umol/L. A Computed Tomography (CT) scan of the abdomen and pelvis was performed, with the differentials considered being incarcerated inguinal hernia, diverticulitis or malignancy. However, the CT showed a $75 \mathrm{~mm}$, contained ruptured right internal iliac artery (IIA) aneurysm, with no active contrast extravasation (Figure 1). There was perianeurysmal haemorrhage in the right iliac fossa which extended into a small fat-containing inguinal hernia (Figure 2). There was also bladder distension and moderate-to-severe right hydroureteronephrosis secondary to the large aneurysm.

Our patient was clinical stable and was therefore planned for endovascular surgery in-hours the next day. He was monitored in Intensive Care Unit overnight, with strict control of systolic blood pressure below $120 \mathrm{mmHg}$ and heart rate below $100 \mathrm{bpm}$, with prazosin and labetalol infusion. An indwelling urinary catheter was inserted to relief the distension and reduce exacerbation of the hydroureteronephrosis.

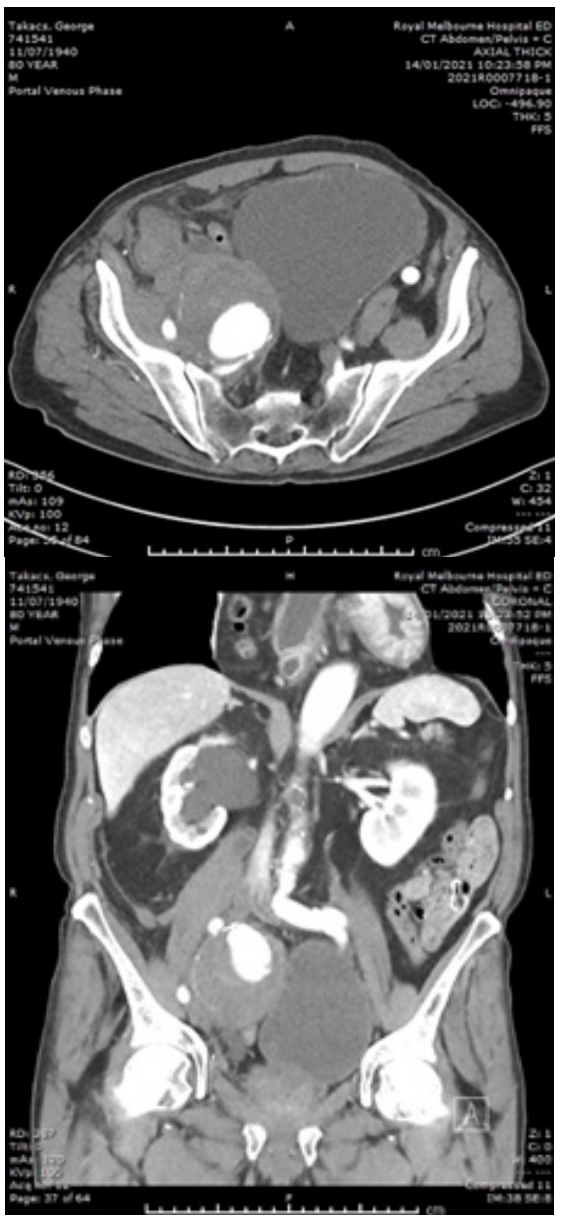

Figure I Arterial-phase CT images of the abdomen and pelvis. (A) Axial image showing the right internal iliac artery aneurysm with contained perforation and no contrast extravasation. (B) Coronal image showing the aneurysm exerting mass effect on bladder, and right sided hydronephrosis. 


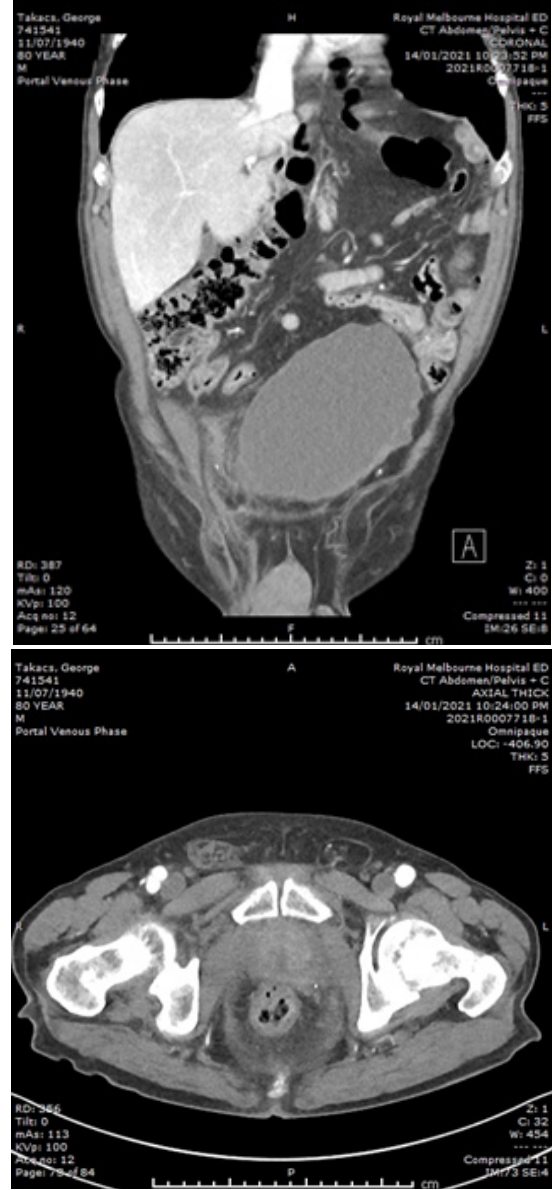

Figure 2 CT images of the abdomen and pelvis. (A) Coronal image showing perianeurysmal haemorrhage in the right iliac fossa extending into an inguinal hernia. (B) Axial image showing the right inguinal hernia containing blood, as compared to left inguinal hernia containing fat only.

The endovascular procedure was performed with accesses via bilateral common femoral artery punctures. Through the left femoral puncture, an up-and-over approach was used to obtain Digital Subtracted Angiograms (DSA), which showed the saccular aneurysm beginning shortly after the right IIA origin, with no other feeding collaterals (Figure 3). Nester embolisation coils and thrombin were deployed and injected to fill the aneurysm. Post-intervention DSA showed very minimal filling of the aneurysm sac. A $13 \times 5 \mathrm{~cm}$ Viabahn covered stent graft was then inserted through the right femoral puncture and deployed over the common and external iliac artery, which covered the origin of the IIA. The groin punctures were closed with Angioseal Closure Devices.

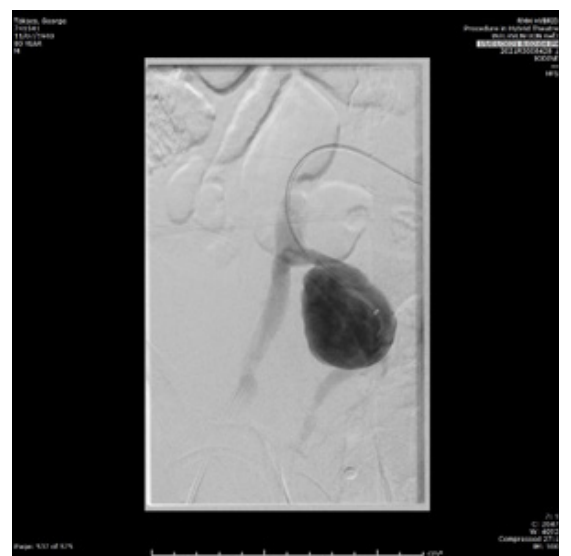

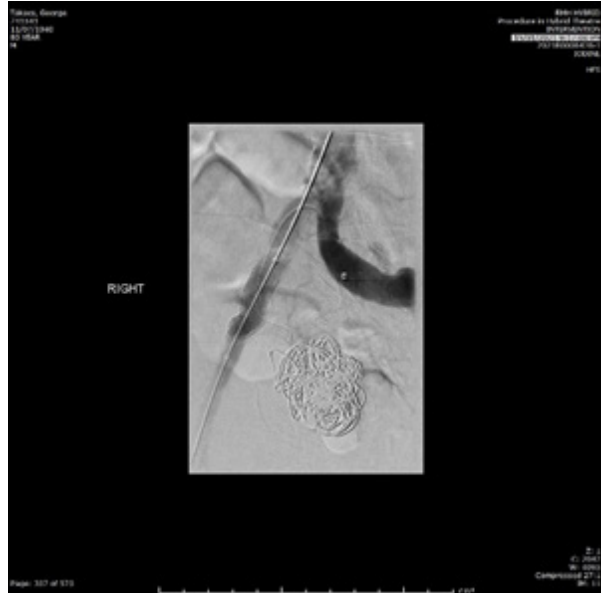

Figure 3 Digital Subtracted Angiograms taken intraoperative pre- and postintervention. (A) The saccular aneurysm is seen filling with contrast shortly after the origin of right internal iliac artery. (B) Successful exclusion of the aneurysm after using embolization coils and thrombin in the aneurysm, and deploying a covered stent graft across the common and external iliac artery.

Our patient had an uneventful recovery, with no puncture complications and remained neurovascularly intact. His abdominal pain resolved, and his groin lump became less symptomatic. His hydroureteronephrosis improved significantly on ultrasound. He had a repeat CT-Angiography, which showed minimal filling of the aneurysm sac, and no other aneurysms in his aorta, iliac and popliteal arteries. Our patient was discharged on lifelong aspirin and planned for ongoing outpatient follow-up.

\section{Discussion}

An aneurysm is defined as a permanent localised dilation in the artery with a diameter at least 1.5 times the normal diameter. ${ }^{1}$ The average IIA diameter is $5.4 \mathrm{~mm}$, and hence an IIA becomes aneurysmal when it is more than $8.1 \mathrm{~mm}$ wide. ${ }^{2}$ The risk factors for IIA are similar to those for AAA, which includes male gender, smoking and hypertension. ${ }^{3}$ In addition, isolated iliac artery aneurysms are rare, representing $0.4-1.9 \%$ of all aneurysms. ${ }^{4}$ There are infrequent case reports describing presentations of solitary IIA aneurysms, which can include symptoms of pelvic organ dysfunction. Pak et al. describes a patient with flank pain due to ureteric obstruction secondary to an aneurysm involving the internal, external and common iliac arteries. ${ }^{5}$ Both ruptured and unruptured IIA aneurysms have been reported to present with urinary retention and obstipation. ${ }^{6,7}$ There was also a case of an unruptured IIA causing symptoms of sciatica due to direct compression of L5 and S1 nerve roots. ${ }^{8}$

IIA aneurysms has high mortality rates of up to $60 \%$ when ruptured. ${ }^{9}$ The important points highlighted in this case was the lack of overt abdominal pain and systemic instability that is usually associated with aortoiliac aneurysm ruptures. To our knowledge, this is the first case where a ruptured IIA aneurysm presents as a reducible inguinal hernia, which only manifested due to the extension of haemorrhage through the hernia sac. There are similar reports of other aneurysms causing symptoms in the groin. Pang et al. reported of an unruptured lateral femoral circumflex artery aneurysm presenting as a painless groin mass. ${ }^{10}$ Ruptured abdominal aortic aneurysms (AAA) has been described to mimic symptomatic groin hernias and testicular pains. ${ }^{11-13}$

This case highlights the importance of considering aortoiliac aneurysms as differentials for abdominal and groin pain or masses. Thorough exploration of the history and careful examination will help flag these diagnoses in our clinical assessment. 


\section{Conclusion}

Patients with IIA aneurysm can have atypical presentations, such as with abdominal masses, groin lumps or vague pelvic organ dysfunction like obstipation and lower urinary tract symptoms. We herein report a case of a ruptured internal iliac artery aneurysm which presented as an inguinal hernia, without any overt abdominal signs or systemic instability. It is crucial to remember that like AAAs, IIA aneurysms carry high mortality when ruptured as well. IIA aneurysms should therefore be an important red flag to rule out in our clinical assessments of patients with such presentations.

\section{Conflicts of interest}

The authors declare no conflict of interest.

\section{Acknowledgments}

None.

\section{Funding}

None.

\section{References}

1. Johnston KW, Rutherford RB, Tilson MD, et al. Suggested standards for reporting on arterial aneurysms. Subcommittee on Reporting Standards for Arterial Aneurysms, Ad Hoc Committee on Reporting Standards, Society for Vascular Surgery and North American Chapter, International Society for Cardiovascular Surgery. Journal of vascular surgery. 1991;13(3):452-458.

2. Horejs D, Gilbert PM, Burstein S, et al. Normal aortoiliac diameters by CT. J comput assist tomogr. 1988;12(4):602-603.
3. Patel NV, Long GW, Cheema ZF, et al. Open vs. endovascular repair of isolated iliac artery aneurysms: A 12-year experience. Journal of vascular surgery. 2009;49(5):1147-1153.

4. Levi N, Schroeder TV. Isolated iliac artery aneurysms. European journal of vascular and endovascular surgery. 1998;16(4):342-344.

5. Pak K, Tomoyoshi T, Tanaka H, et al. Ureteral obstruction secondary to iliac artery aneurysm. Hinyokika kiyo Acta urologica Japonica. 1988;34(3):496-501.

6. Guru KA, Sarle RC, Reddy D, et al. Iliac Artery Aneurysm: A Fatal Cause of Urinary Retention. J endourol. 2003;17(4):221-222.

7. Musić D, Radević B, Batrićević G, et al. Isolated aneurysm of the internal iliac artery and disorders of the pelvic organs functions. Vojnosanitetski pregled. 2007;64(5):357-360.

8. Vascular Surgery Program Abstracts. ANZ Journal of Surgery. 2009;79(1):213-222.

9. Sandhu RS, Pipinos. Isolated iliac artery aneurysms. Seminars in vascular surgery. 2005;18(4):209-215.

10. Pang JH, Elbasty A, Meyer FJ. Curious case of a true aneurysm of the lateral femoral circumflex artery: a rare but important differential in the diagnosis of a painless groin mass. BMJ Case Rep. 2019;12(3):e228312.

11. Moissinac K, To B, Liew N, et al. Abdominal aortic aneurysm rupture masquerading as strangulated inguinal hernia. The American journal of emergency medicine. 2001;19(7):604-605.

12. Klein HJ, Becker D, Rancic Z. Diagnosis and perioperative management of ruptured AAA mimicking symptomatic groin hernia. Int J Surg Case Rep. 2016;18:1-4.

13. Khan T, Mahomed A, Ghoorun B, et al. Acute testicular pain secondary to a leaking abdominal aortic aneurysm (AAA). BMJ Case Rep. 2018;2018:bcr-2018-226577. 